\title{
History note: tragedy of Thorotrast
}

\author{
Shoichi Takekawa ${ }^{1,2} \cdot$ Yoshihiko Ueda $^{3}$ - Yoshihiro Hiramatsu ${ }^{2}$. \\ Kihachiro Komiyama ${ }^{2} \cdot$ Hirotsugu Munechika $^{1}$
}

Received: 16 August 2015 / Accepted: 2 September 2015 / Published online: 29 September 2015

(C) Japan Radiological Society 2015

\section{Introduction}

Learning from the history is something that we should continue over the generations. Thorium dioxide suspension, trade name "Thorotrast", used to be considered an apparently innocent contrast medium that was used for angiography with adequate radiodensity and no significant acute or subacute adverse reactions at the clinical standard in the past. However, it was an alpha emitter which was deposited in the reticuloendothelial system, including the liver, spleen and lymph nodes, and it irradiated such organs for a lifelong period [1-16]. Presented here is a tragic case that may be known to radiologists of older generations, but such experiences may not have been shared by younger radiologists, since almost all victims of Thorotrast injection are now dead. Although we will not see this type of case any more, we believe we should document historically important issues that may help shape our future practice.

Dr. Kihachiro Komiyama deceased (contribution to survey of the literature and advice).

Shoichi Takekawa

takekawasd@mt.strins.or.jp

1 Department of Diagnostic Radiology, Southern Tohoku General Hospital, 7-115, Yatsuyamada, Koriyama, Fukushima 963-8563, Japan

2 Formerly Department of Radiology, Dokkyo Medical University Koshigaya Hospital, 2-1-50, Minamikoshigaya, Koshigaya, Saitama 343-8555, Japan

3 Department of Pathology, Dokkyo Medical University Koshigaya Hospital, 2-1-50, Minamikoshigaya, Koshigaya, Saitama 343-8555, Japan

\section{Case}

A 69-year-old man presented with sudden onset of pain in the right upper abdomen. An enlarged liver and jaundice were found by his family physician and he was referred to our University Hospital. In 1936, 49 years previously, he underwent angiography of the left forearm with a contrast medium of undocumented type for evaluation of fracture in a hospital of the Imperial Army. Laboratory data revealed elevated bilirubin (total $3.8 \mathrm{mg} / \mathrm{dl}$, direct $2.7 \mathrm{mg} / \mathrm{dl}$ ), elevated liver enzyme (AST 215KU, ALT $93 \mathrm{KU}$ ), anemia (RBC $332 \times 10^{4}$, Hb $11.0 \mathrm{~g} / \mathrm{dl}$, WBC 8600), platelet count $23.6 \times 10^{4}$, negative AFP, negative HBsAg and HBsAb, total protein $5.7 \mathrm{~g} / \mathrm{dl}$, erythrocyte sedimentation rate $22 / \mathrm{h}$, and serum creatinine $1.6 \mathrm{mg} / \mathrm{ml}$. On physical examination a small amount of ascites was noted. The liver was enlarged and palpated 3 finger-breadths below his right costal margin. The patient expired soon after radiography and CT with no further examinations. Radiography of the abdomen revealed enlarged liver and atrophic spleen containing radiopaque material (Fig. 1). Non-enhanced CT of the upper abdomen showed a large low-density mass in the liver, containing irregular streaky densities. Reticular high densities were also seen in the other areas of liver parenchyma. There were spotty increased densities at the hepatic port and around the pancreas. The spleen was markedly dense and atrophic, consistent with Thorotrast deposition (Fig. 2a-c). CT of the abdomen after administration of contrast medium showed irregularly enhanced areas in the round low-density mass in the right lobe of the liver, consistent with a solid neoplasm (Fig. 3d, e). There was also a moderate amount of ascites around the liver (Fig. 3f). 
Fig. 1 Plain radiographs of the abdomen. a The liver is enlarged and increased in density. The right costophrenic angle is obscured. There is an increased density in the region of the spleen (arrow). b The increased density in the spleen suggests Thorotrast deposition in the atrophic spleen (arrow). The "dog's ear sign" in the pelvic region is suggestive of ascites

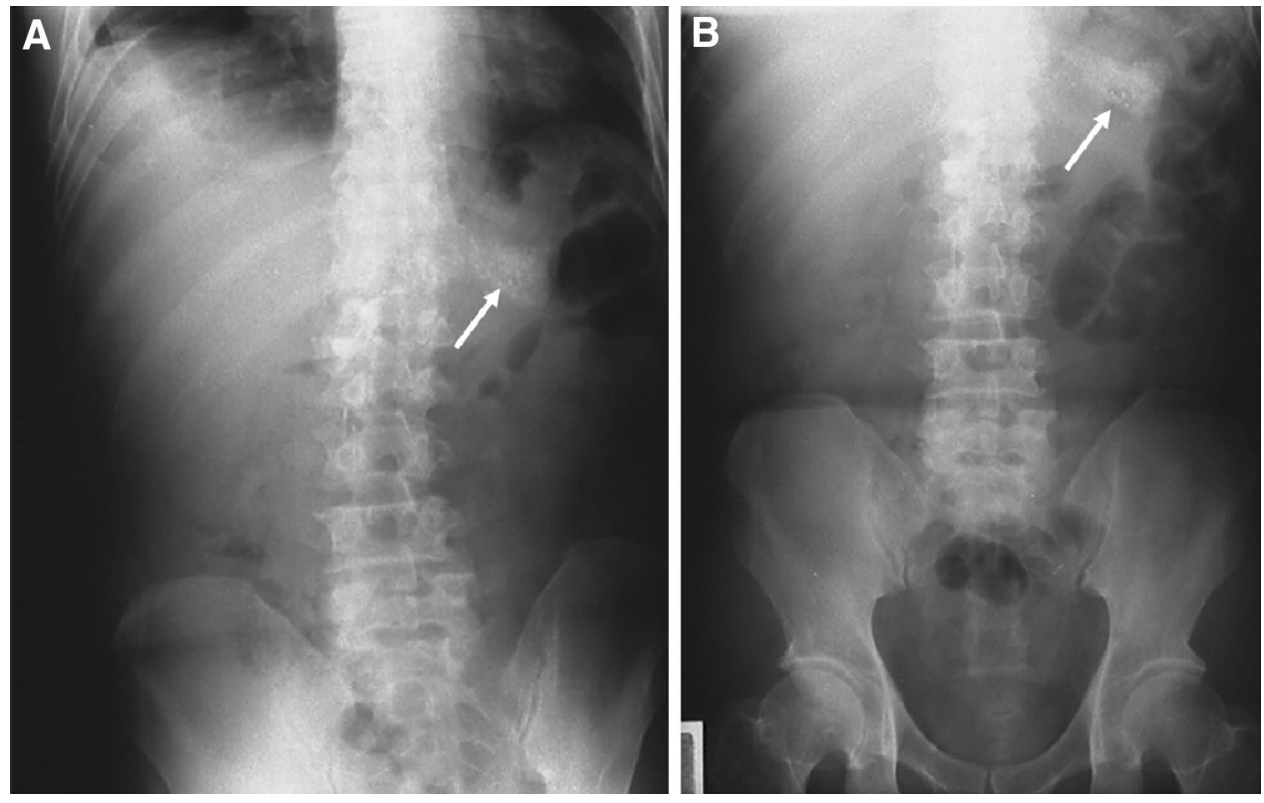

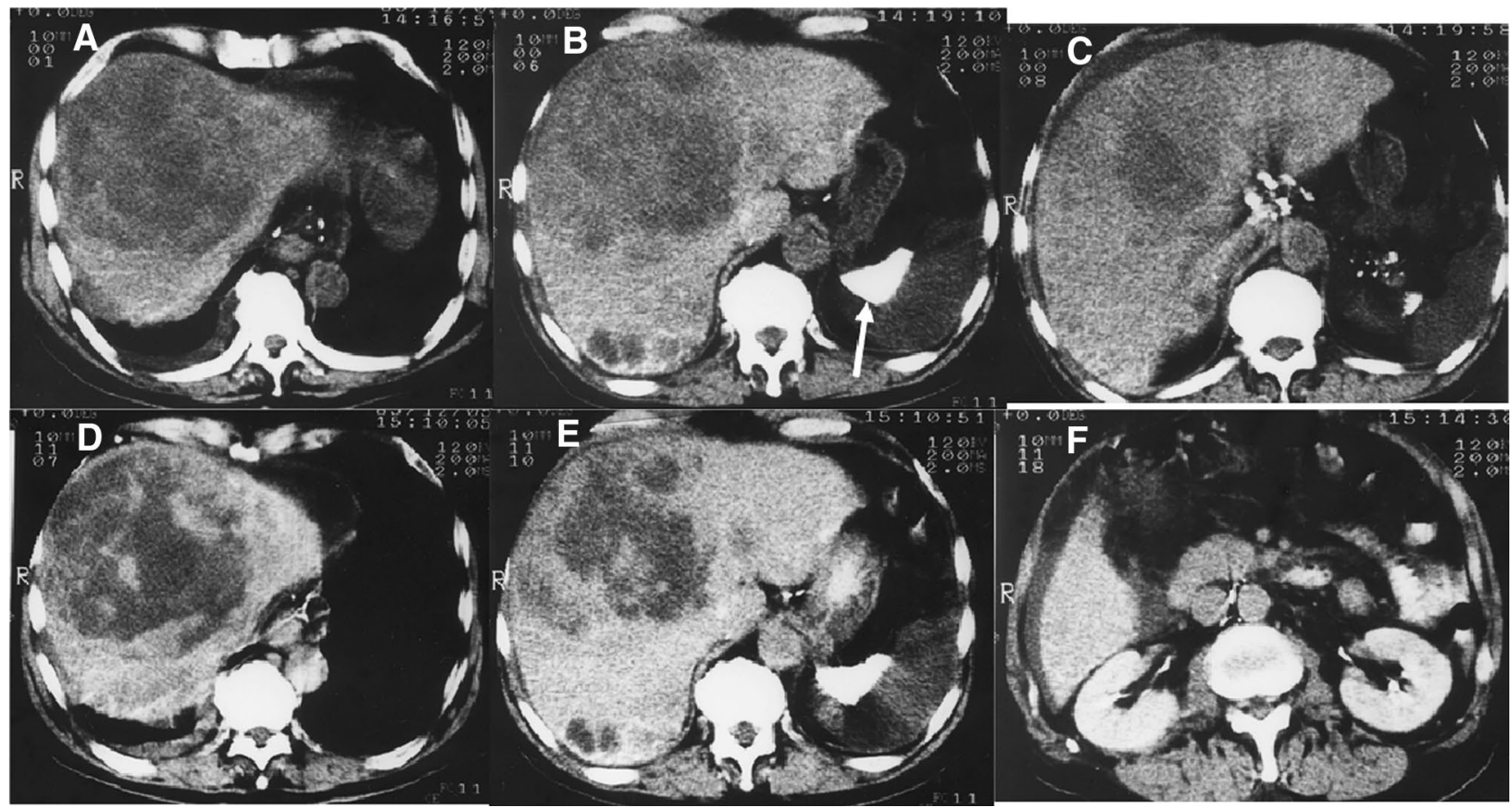

Fig. 2 CT of the liver. a-c Non-enhanced CT of the upper abdomen shows a large round low density mass, containing some irregular streaky densities inside the mass, in the right lobe of the liver, and scattered small low density spots in both lobes of the liver. In general, there are reticular high-densities in the parenchyma of the liver. Spotty high-densities are noted at the porta hepatis and around the pancreas. The spleen (arrow in b) is markedly dense and atrophic, consistent with Thorotrast deposition. d, e CT of the abdomen after intravenous administration showed irregularly enhanced foci in the round mass, consistent with a liver neoplasm. f Moderate amount of ascites is seen around the liver

patient's liver weighed much more than normal, because the liver harbored a tumor.

There were esophageal varices. Ascites, $600 \mathrm{ml}$, was hemorrhagic. The spleen weighed $12 \mathrm{~g}$, and was fibrosed spongy. The average weight of the liver in Japanese adults is $1262 \mathrm{~g}$ in men and $1147 \mathrm{~g}$ in women. Therefore, the 
Fig. 3 Radiography of autopsy specimens. a Liver. There are reticular high-densities in the liver parenchyma. Very highdensities are noted at the porta hepatis, suggestive of Thorotrast deposition in the lymph nodes. b Pancreas and spleen. Heavily increased densities are noted in the pancreatic head and atrophic spleen (arrow)

Fig. 4 Photomicrography. a Angiosarcoma of the liver. b High-power magnified image of angiosarcoma of the liver. c Deposition of Thorotrast (brown particles) in the liver. d Deposition of Thorotrast in the spleen
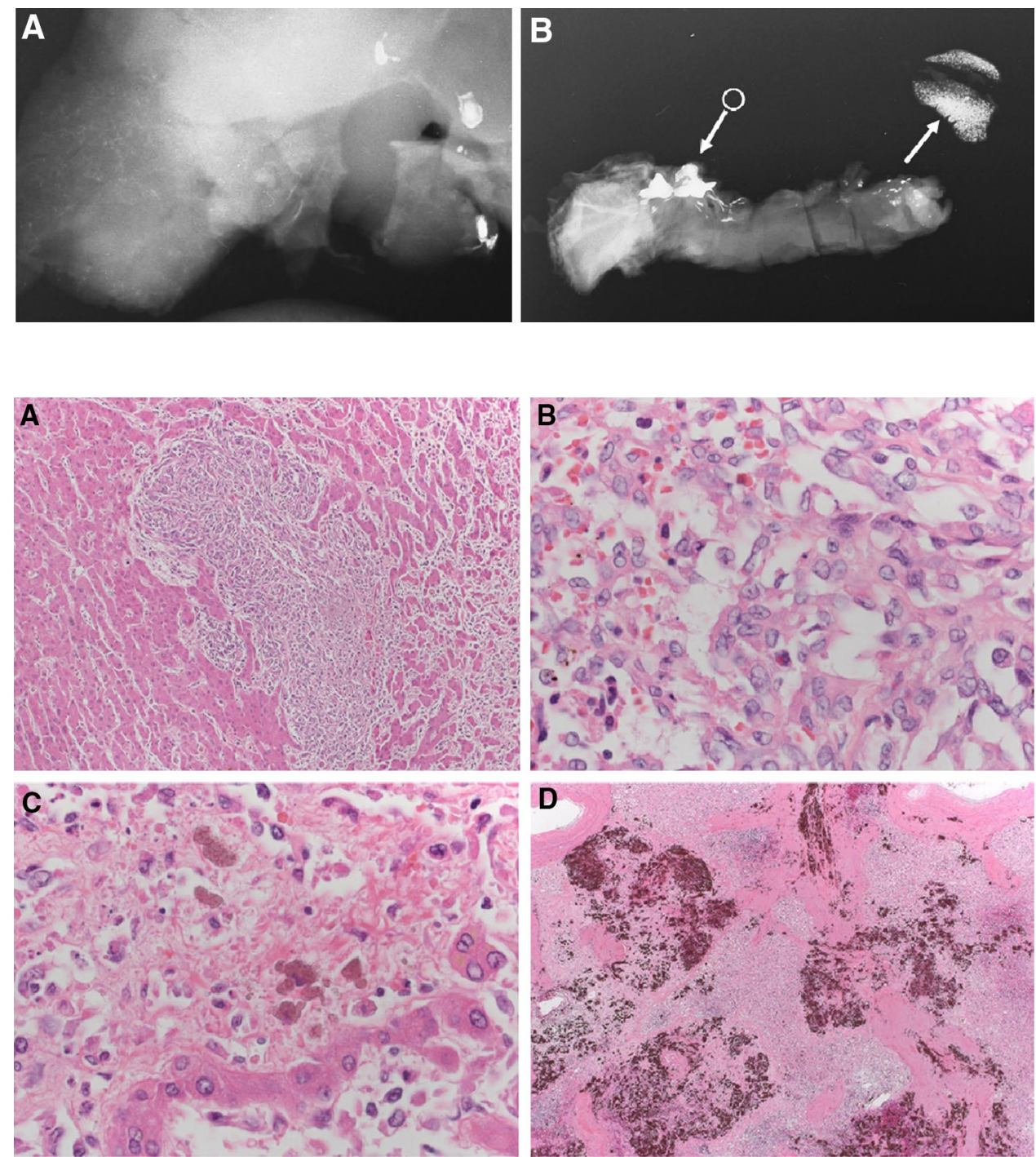

and atrophic. The average weight of the spleen in Japanese adults is $131.5 \mathrm{~g}$ in men and $122.4 \mathrm{~g}$ in women. Therefore, the spleen was much smaller than normal.

There were reticular high densities in the liver parenchyma on organ radiographs. There were increased densities around the porta hepatis and pancreas, suggesting Thorotrast deposition in the lymph nodes. There were heavily increased densities in the region of the pancreatic head and also in the atrophic spleen (Fig. 4). Photomicrographs of the liver showed irregular and diffuse growth of tumor cells, which were large endothelial cells with atypia (Fig. 4a: low-power magnification, b: high-power magnification). Deposition of Thorotrast was seen as brown particles in the liver (Fig. 4c). Heavy deposition of Thorotrast was seen in the spleen (Fig. 4d). Immunohistochemistry of the tumor cells of the liver revealed positive CD31 and CD34 (Fig. 5a, b). Pathological findings were consistent with angiosarcoma secondary to long-standing deposition of Thorotrast. The specimen sections of the liver were also studied for radioactivity of thorium alpha particles by autoradiography at Aichi Cancer Center, courtesy of Dr. Choichiro Kido, Director at the time. The autoradiography proved the activity of alpha particles (not shown). The concentration of Thorotrast in the liver was measured as $0.353 \mathrm{mg} / \mathrm{g}$.

\section{Discussion}

Thorotrast is a trade name of radioactive thorium dioxide suspension, widely used in the 1930s and 1940s. It was considered to be an ideal arteriographic contrast medium due to its high radiodensity and relatively low acute and subacute complication rates. Its distribution in the body depends on the route of administration, but, if administered through an intravascular route, it stays in the reticuloendothelial system 
Fig. 5 Immunohistochemistry. a CD31 was positive. b CD34 was positive
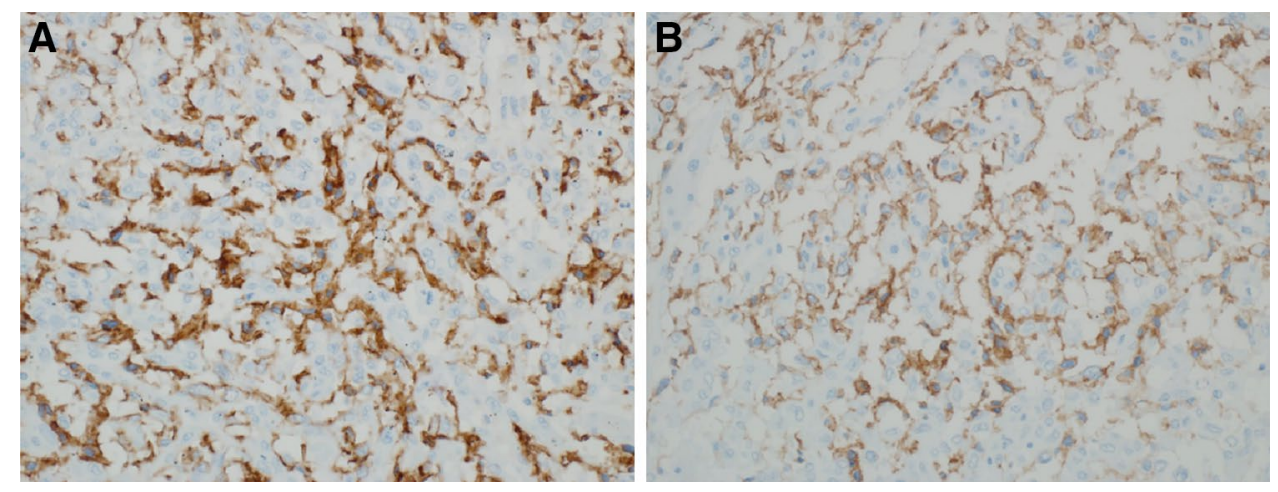

with slow alpha decay (thorium-232 $\rightarrow$ radium-228 $+\alpha$; physical half-life $1.405 \times 10^{10}$ years; biological half-life estimated to be 400 years) $[17,18]$. It was used widely for 2-10 million people in the world, and for 10-20 thousand people in Japan, mainly in Imperial Army hospitals [6-8].

The case presented developed angiosarcoma of the liver, 49 years after Thorotrast injection. Malignancy associated with Thorotrast deposition is various, including malignancy in the liver, biliary tracts, hematopoietic system, lymphoma, lung, peritoneum, bone and others [116]. According to Ito and his associates [15] the hepatic tumors after Thorotrast deposition comprised cholangiocarcinoma (CCA) (44 cases, $43.1 \%$ ), angiosarcoma (AS) (39 cases, $38.3 \%$ ), hepatocellular carcinoma (HCC) (16 cases, $15.7 \%)$, triple cancers (CCA + AS + HCC) (1 case, $1 \%)$, and double cancers (CCA + HCC) (2 cases, $2 \%)$. Angiosarcoma of the liver, rare in the general population, is a relatively specific tumor after Thorotrast deposition.

Weber et al. [19] reported in 1995 about their experience of seeing a female patient with sudden right-sided abdominal pain. Subsequently, hepatic carcinoma induced by Thorotrast exposure was found in her body. The patient did not remember that she had received an injection of a contrast medium like Thorotrast. They concluded in saying, "This case leads us to remind recently trained clinicians of this historical pathology, which obviously will disappear with time, but must kept in mind for at least two decades, when confronted with atypical abdominal pain in elderly patients."

This is a lesson in the necessity of long-term follow-ups for a specific study or treatment, learned from an article on Thorotrast.

\section{Conclusion}

Thorotrast seemed to be an ideal contrast medium at the beginning, but keen research followed and studied the effects of long-standing radiation from Thorotrast.
In fact, several researchers $[4,17]$ warned radiologists to withhold or stop the use of Thorotrast and to continue the follow-up of previously studied Thorotrast recipients.

What we learn from old cases like the one we presented in this paper would be that we should continue to follow and study the sequences and results of drugs, contrast medium and techniques.

Acknowledgments The authors thank Choichiro Kido, M.D., then Vice President of Aichi Cancer Center Hospital, for his help in performing autoradiography for alpha tracks and the measurement of concentration of Thorotrast. A part of this case was presented at the 22nd Autumn Assembly of Japan Radiological Society in 1987. The authors also would like to acknowledge editorial assistance by Dr. Shigeru Ehara, Professor of Radiology, Iwate Medical University.

\section{Compliance with ethical standards}

Conflict of interest The authors declare that they have no conflict of interest.

\section{References}

1. MacMahon HE, Murphy AS, Bates MI. Endothelial-cell sarcoma of liver following Thorotrast injections. Am J Pathol. 1947;23:585-611.

2. Kakei H, Sugimura T, Nakao K, Imura H. Radioautography of Thorotrast-deposited organ. Nippon Acta Radiol. 1952;22:479.

3. Plenge K, Krückenmeyer K. Über ein Sarkom am Ort der Thorotrastinjektion. Zblt Allg Path. 1954;92:255-60.

4. Budin E, Gershon-Cohen J. The danger of cancer from Thorotrast as a diagnostic medium. AJR Am J Roentgenol. 1956;75:1188-93.

5. Horta da Silvia J. Malignancy and other late effects following administration of Thorotrast. Lancet. 1965;2:201-5.

6. Takahashi S, Kitabatake T, Yamagata S, Miyakawa T, Masuyama M, Mori T, et al. Statistical study on Thorotrast-induced cancer of the liver. Tohoku J Exp Med. 1965;87:144-54.

7. Mori T, Nozue Y, Okamoto T, Tanaka T, Sugita K, Tsuda T. Follow-up study of the fate of patients who had been administered Thorotrast more than 22 years previously. Nippon Acta Radiol. 1966;25:1144-65 (in Japanese).

8. Mori T, Sakai T, Nozue Y, Okamoto T, Wada T, Tanaka T, et al. Malignancy and other injuries following Thorotrast administration: follow-up study of 147 cases in Japan. Strahlentherapie. 1967;134:229-34. 
9. Abo M, Sugisawa T, Shindo K, Soezima Y, Ichimura H. Cancer of the liver following Thorotrast injection. Gan no Rinshou (Clinical Practice of Cancer). 1969;15:822-6 (in Japanese).

10. Ishida H, Inoue S, Komatsu M, Mukojima T, Awatsu R, Ashida $\mathrm{M}$, et al. Computed tomographic findings in Thorotrast recipients. KANZO (Liver). 1982;23:297-306.

11. Sadahira Y, Mori M, Nakamoto S, Awai M, Mori T. Lung cancer in a Thorotrast administered patient. Acta Pathol Jpn. 1985;35:1467-73.

12. Hirakawa S, Sasaki C, Sasaki Y, Eto T, Kawabe H, Hagino S, Moriyama Y. Search for retained contrast medium containing radioactive ${ }^{232} \mathrm{ThO}$, which was administered about 48 years ago. J. Mutsu Gen Hosp. 1987;3:1-8.

13. Kido Ch, Barron JP, editors. Thorotrast and carcinogenesis: basic and clinical studies. Report of researches supported by grants from Ministry of Health and Welfare of Japan, March, 1989. p. $1-184$.
14. Gv Kaick, Dalheimer A, Hornik S, Kaul A, Liebermann D, Luehrs H, et al. The German Thorotrast study: recent results and assessment of risks. Radiat Res. 1999;152:S64-71.

15. Ito Y, Kojiro M, Nakashima T, et al. Pathomorphologic characteristics of 102 cases of Thorotrast-related hepatocellular carcinoma, cholangiocarcinoma and hepatic angiosarcoma. Cancer. 1988;62:1153-62.

16. Fukumoto M. Radiation pathology: from Thorotrast deposition. Pathol Clin. 2011;29:761-8 (in Japanese).

17. Janower ML, Miettinen OS, Flynn MJ. Effects of long-term Thorotrast exposure. Radiology. 1972;103:13-20.

18. Kaplan AW, Teng SS, Koo AH. CT recognition of Thorotrast-induced intracranial and lumbar arachnoiditis. AJNR. 1984;5:323-5.

19. Weber E, Laarbaui F, Michel L, Donckier J. Abdominal pain: do not forget Thorotrast! Postgrad Med J. 1995;71:367-8. doi:10.1136/pgmj.71.836.367. 